\title{
Modes of pointing to existing spaces and the use of frames of reference
}

\author{
Olivier Le Guen \\ Max Planck Institute for Psycholinguistics, Nijmegen / CIESAS, Mexico City
}

\begin{abstract}
This paper aims at providing a systematic framework for investigating differences in how people point to existing spaces. Pointing is considered according to two conditions: (1) A non-transposed condition where the body of the speaker always constitutes the origo and where the various types of pointing are differentiated by the status of the target and (2) a transposed condition where both the distant figure and the distant ground are identified and their relation specified according to two frames of reference (FoRs): the egocentric FoR (where spatial relationships are coded with respect to the speaker's point of view) and the geocentric FoR (where spatial relationships are coded in relation to external cues in the environment). The preference for one or the other frame of reference not only has consequences for pointing to real spaces but has some resonance in other domains, constraining the production of gesture in these related domains.
\end{abstract}

Keywords: pointing, frames of reference, geocentric gesture, Yucatec Maya

\section{Introduction}

How can gesture help us to understand communication processes and mental representations? Recently, numerous studies have specifically looked at the semantic complementarity of speech and gestural modalities as well as the extent to which gestures carry information in face-to-face communication (Clark, 1996; Enfield, 2009; Engle, 1998; Goodwin, 2000; Haviland, 1993; Kelly et al., 2009; Kendon, 2004; Liddell, 2003; McNeill, 1992; Okrent, 2002; Schegloff, 1984). In the spatial domain, one issue concerns how gesture reflects the preference for particular frames of references in various speech communities (Brown \& Levinson, 2009; Haviland, 1996, 2000; Kita, 1998; Levinson, 2003). Among the Yucatec Maya of Mexico, for instance, Le Guen (2011) proposes that gesture is the medium through which spatial frames of reference can be learned and sustained in interaction. 
While the majority of research concerned with gesture and space has looked at Western speakers, the few cross-cultural studies dealing with this issue have often been limited to pointing gestures (Enfield et al., 2007; Kita, 2003b; Levinson, 2003). Even fewer studies have dealt with the impact of the geocentric frame of reference on gesture production in genral and in pointing in particular (Haviland, 1993, 1996, 2000; Levinson, 2003). This paper aims to contribute to filling this gap by providing a framework for systematically investigating differences in spatial gesture production in non-transposed context (pointing for the current position of the speaker) and in transposed context (pointing to distant figure and ground), according to two frames of reference (FoRs): the egocentric FoR (where spatial relationships are coded with respect to the speaker's point of view; e.g., 'left' and 'right') and the geocentric FoR (where spatial relationships are coded in relation to external cues in the environment; e.g., 'north' or 'south').

Pointing has been the subject of much investigation. However, this term has been used to refer to a wide range of phenomena. This paper will use the definition provided by Enfield et al. (2007) who define pointing "as a communicative bodily movement which projects a vector whose direction is determined, in the context, by the conceived spatial location, relative to the person performing the gesture, of a place or thing relevant to the current utterance." In this paper, the body of the speaker will be treated using the more general notion of 'origo' (Bühler, 1983; Hanks, 1990). Pointing is a process that encompasses several features: an origo, a target and a vector that encodes a specific direction determined by the anchor point. A schematization of pointing is presented in Figure 1.

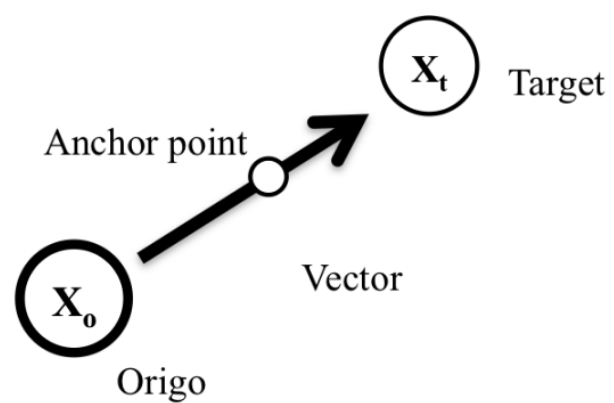

Figure 1. The features of pointing

The origo is the source of the vector. The vector is a semi-axis whose orientation is determined by the anchor point (see Levinson, 2003). In pointing, the anchor point is not on the body but is on the extended arm or finger that specifies the orientation of the vector. Because gestures are produced in 3D space, the orientation of the vector can be calculated in accordance with a $x$ and a $y$ axis: (1) an $x$ axis or horizontal plane of 360 degrees around the body of the speaker and (2) a $y$ or a 
vertical plane of 180 degrees starting from the feet of the speaker and ending at the space above his head. The target is the referent of the pointing.

Crucial to the analysis of pointing to existing space (i.e., real places in the world) is the process of transposition. In the non-transposed condition, the body is always the origo and therefore only the target is to be determined. In contrast, under transposition, the body of the speaker will represent two origos at the same time: the origo starting from the body during the speech production (i.e., in the Speech Event) that stands for a projected origo in the depicted spatial scene (i.e., in the Narrated event). Only under the transposed condition are the frames of reference involved because only in this condition has the figure (i.e., the target) to be located in relation to a ground that is not the body.

The various types of pointings explored in this paper are summarized in Figure 2. Note that the term pointing is used here based on a definition that considers its meaning (i.e., the orientation of the gesture), and not its morphology (e.g., what kind of hand shape is used) following for instance Kendon and Versante (2003) or Kendon (2004, chap. 11).

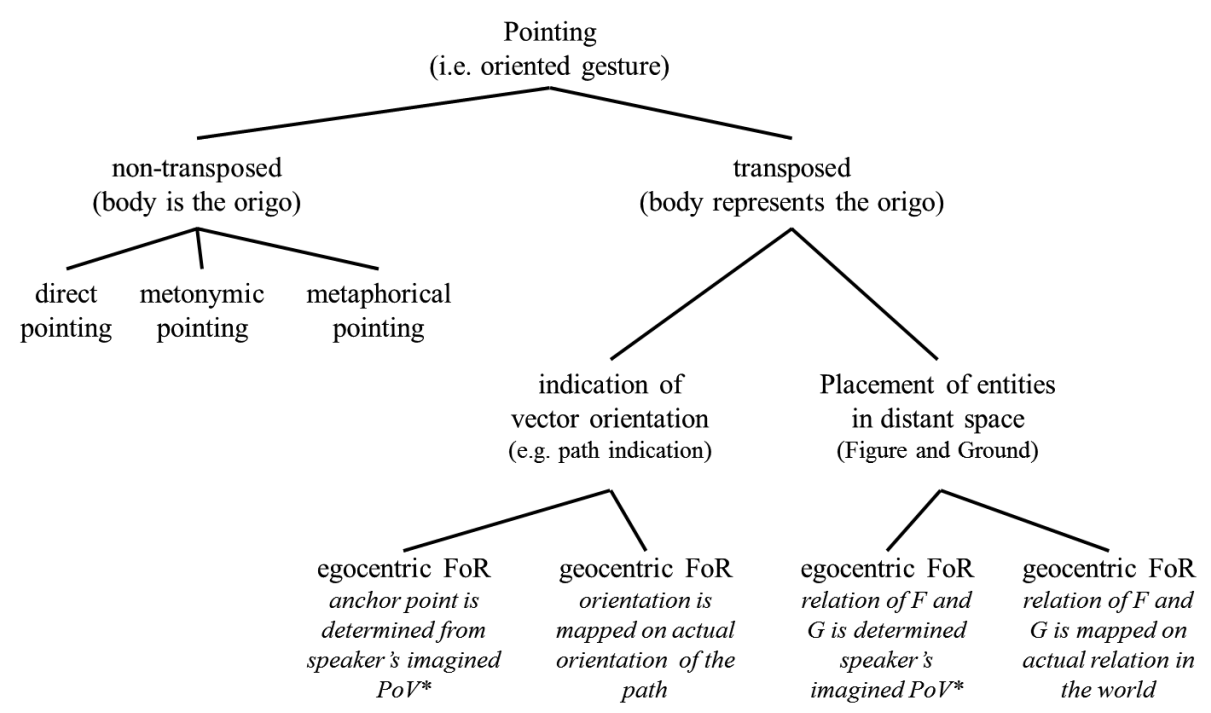

Figure 2. The various types of pointings considered in the paper $\left({ }^{\star} \mathrm{PoV}\right.$ : Point of View)

\section{Frames of reference}

A Frame of Reference (FoR) can be defined according as a coordinate system used to localize a figure with respect to a ground. Levinson (2003) summarizes the literature and considers three types of frames of reference: the 'intrinsic', the 'relative' and the 'absolute.' Although Levinson's proposal about these three frames is 
adopted in this paper, his terminology will not be followed. Instead, 'egocentric' is substituted for 'relative' and 'geocentric' is used for 'absolute.' The definitions of FoRs used in this paper are logical (i.e., defined in terms of the sufficient information they provide a person to be able to locate a figure in relation to a ground) and not only linguistic as in Levinson and colleagues proposal.

The intrinsic frame of reference defines the location of a figure by using the internal divisions or parts of the Ground object - its front(s), back(s) and side(s) - to establish an axis for locating the Figure. In the intrinsic FoR there are only two terms involved: the figure and the ground. What determines the search domain will be an anchor point placed on the ground (on its front, back or side, etc.). Note that if the ground rotates the relation is modified. It is important to distinguish intrinsic FoR from deixis, especially when pointing is involved. Although in both cases the body can be considered as the origo, only in the intrinsic FoR is the body the ground which internal division will matter to locate the figure. For instance, in an utterance like "the kettle is in front of me" it is the internal division of the speaker's body that will determine the search domain where the figure will be located. On the other hand, in the following deictic utterance, "the kettle is over there," the internal division of the body is irrelevant (the speaker can point facing or not the kettle), and some additional information should be considered, such as the relative distance to the referent or the perceptual access of the interlocutor (see Hanks, 1990, 2005). Additionally, a pointing can be performed under transposition (gesturally or with eye gaze) but, crucially, in this condition the body is not the ground, only the origo. This analysis differs from Danziger (2010a)'s proposal of reducing gestural deixis to what she proposes as the 'direct FoR.' In real life direction giving or describing the placement of distant entities (i.e., transposed conditions when the origo is not the body), the intrinsic FoR is not precise and therefore not useful on its own. Imagine a situation where a person A wants to borrow person B's car. If B limits herself to tell A that her car is parked on the 'side of the church', A would have no idea which side of the church the car might be. Additional information is required in cases where the ground's internal division is not obvious or nonexistent (e.g., a tree for instance).

The egocentric frame of reference encodes the location of a figure with respect to a ground on the basis of the point of view of the speaker. The egocentric FoR is a three term relation where the point of view of the speaker imposes the anchor point onto a ground from which to project the search domain where the figure is located. Crucially, in the egocentric FoR the body is never the ground and always external to the scene (i.e., the ground and the figure). Note that if the speaker changes position (e.g., by moving around the ground) the relation is modified (e.g., what was on the left of the speaker will be on the speaker's right). The use of the egocentric FoR by a speaker requires the interlocutor to know the speaker's 
orientation, that is, to imagine the scene through the eyes of the speaker as the speaker imagines it when viewing the scene, a process called 'projection.' Imagine the same situation where A wants to borrow B's car. B cannot simply answer that her car is to the left of the church. She needs to give a cue regarding her imaginary position in relation to the scene, saying something like: "looking at the entrance of the church [the car is to the left of the church]."

The geocentric frame of reference specifies the location of a figure in relation to a ground in terms of fixed extrinsic angles. This geocentric FoR corresponds in many societies to the use of cardinal directions in describing spatial relations both in large spaces as well as small-scale tabletop scenes, and it can involve a conceptual 'slope' (an uphill/downhill or up/down river opposition for instance). The geocentric FoR implies a ternary relation. However, although extrinsic to the ground, the anchor point determines a search domain from the ground. Imagine again the situation where A wants to borrow B's car. If B uses a geocentric FoR, she can simply answer that her car lies 'on the north side of the church.' No matter where A is coming from, there is only one search domain determined by the absolute anchor point: the cardinal direction north. In contrast with the egocentric FoR, a speaker using the geocentric FoR does not have to give any cue regarding the orientation of their viewpoint on the scene. Moreover, the examples of geocentric gestures to be given below will show that even verbal cues (e.g., the use the word "north") can be spared.

\section{The aim and the scope of this paper}

This paper deals exclusively with pointing about existing places, that is locations of real entities in the world (e.g., directly visible referents but also cities, landmarks, houses, natural formations, etc.) and real path indications. These types of referents contrast with notional or imaginary spaces created in the gestural space of the speaker during a telling for instance. Two types of gesture are considered: path indication and placement of entities in (geographical) remote space.

Some definitions of pointings in non-transposed and transposed conditions are proposed (most of them taken from the existing literature) and illustrated with concrete examples from data collected by the author. The examples provided come from two speech communities: Yucatec Maya (Mexico) and French (France). Several types of data are examined: natural conversation, elicitation and experimental (Le Guen, 2006, 2009, 2011). Because the issue of pointing to real space relies on the logical properties of the use of space and on communicative constraints, only qualitative examples are examined. Indeed, most of the problem involved in the use of the frames of reference actually relies on what McNeill (2003) has referred to as the 'morality' of pointing, i.e., what counts as a pointing that entails some 
kind of communicative truth. Haviland (2000) also deals with this issue but from a linguistic-anthropological perspective and concentrates on the social construction of shared knowledge when gesturing in relation to existing places but without offering a systematic typology of pointing.

This paper proposes a set of definitions that exhibit the logical properties of each kind of pointing as well as their communicative constrains. Central to this paper is how the speakers' preference for the egocentric or the geocentric FoR under transposition has logical consequences for pointings in the non-transposed condition but also in other domains (e.g., time). Two groups of speakers that display some tendencies in the use of pointing to real space are considered: the 'egocentric coders' (who use primarily the egocentric FoR under transposition) and 'geocentric coders' (who use primarily the geocentric FoR under transposition). If the consequences of the preference for one specific FoR affects the cognitive representation of space (Levinson, 2003), this preference is primarily guided by communicative and interactional constraints.

One issue with gesture that the reader should keep in mind, as presented in more detail below, is that gestures that look alike can be (especially cross-culturally) easily (wrongly) interpreted by a viewer for their meaning are not always identical. This is the reason why, when dealing with a pointing gesture done in accordance to FoRs, a test of rotation should be performed in order to disambiguate which FoR is used in the production of the gesture.

\section{The Yucatec Maya setting}

Since many of the examples presented in this paper are taken from the Yucatec Maya, this section briefly introduces the reader to the Yucatec Maya ethnographic setting.

Yucatec Maya belongs to the Yucatecan branch of the Maya family and is spoken in Southern Mexico (in the states of Yucatán, Campeche and Quintana Roo) and in Northern Belize. The data presented in this paper were collected from the village of Kopchen where the author has been conducting fieldwork on a regular basis since 2002. The village is situated in the state of Quintana Roo in the so-called "Zona Maya," an area which has preserved many aspects of traditional Maya life and where the Yucatec Maya language is still widely spoken; indeed, women over 40 years old are monolingual in Yucatec Maya. Younger generations receive some schooling in Spanish but everyday interactions in the village are carried out in Maya.

The village is surrounded by tropical forest. The terrain is flat and vertical relief never exceeds a few meters. In central Quintana Roo, Yucatec Maya still perform slash-and-burn subsistence agriculture. Ethnographic information and further references on this area can be found in Le Guen (2006). 


\section{Types of pointing in non-transposed settings}

In non-transposed settings, the origo is always the body of the speaker and no frames of reference are involved. ${ }^{2}$ Different types of pointing (direct, metonymic and metaphoric) are defined by the target considered.

\section{Direct pointing}

In direct pointing the origo is the body of the speaker and the extended arm or finger (or in some cases the gaze, eyebrow or lips) gives the orientation of the vector (i.e., determines the anchor point) towards the target. Direct pointing to actual places involves two features: (1) the arm is oriented according to the accurate angle with respect to the target (i.e., the actual place occupied by the entity in the world) and (2) the target or its location are directly cognitively available to speakers (even if not directly visible). This type of pointing (usually referred in the literature solely as 'pointing') is the one used by preverbal children and great apes and is considered universal (Tomasello, 2008).

In example 1, a Yucatec Maya speaker (W.) is telling the author about the journey of the Saint of her village (a ritual event that takes place every year). During this ritual journey, the Saint left the village and went to visit several surrounding villages. Every time the speaker refers to a place the Saint visited, she points accurately towards the place she mentions in her speech. In example 1 , the speaker points to two locations. The first is the church from where the saint is leaving for his journey (Figure 3a). The church is located only a few meters from W.s current position (her house) to the north, while $\mathrm{W}$. is oriented towards the east. The pointing that follows refers to the third stop of the Saint in Hazil, a village situated 12 $\mathrm{km}$ to the east (Figure $3 \mathrm{~b}$ ). W. now points to the north east and a little more upward (to indicate that the target is more spatially distant). In her utterances, each place name is accompanied synchronically with a pointing gesture performed accurately in terms of angular information towards the target.

(ex.1) poos le k'n luk'uk [waya']... [Ha'asil]

$$
\text { 'Well, when (the Saint) leaves }\left[(\text { from) here }]_{\text {Figure 3a }} \ldots \text { (it's) }[\text { Hazil }]_{\text {Figure 3b }}\right.
$$

In this example, no matter what distance away the target is (if it is close and directly visible as in the case of the church or several kilometers away as in the case of Hazil), the speaker always performs her pointing towards the actual location of the target. ${ }^{3}$ During my fieldwork experience in the village, I often have been asked to point to my home place (France). My Maya interlocutors explicitly wanted to know the correct orientation of France, probably for future conversations in order to be able to point to it in case they are mentioning this place and, inversely, to understand a potential gestural reference to this target produced by me. 


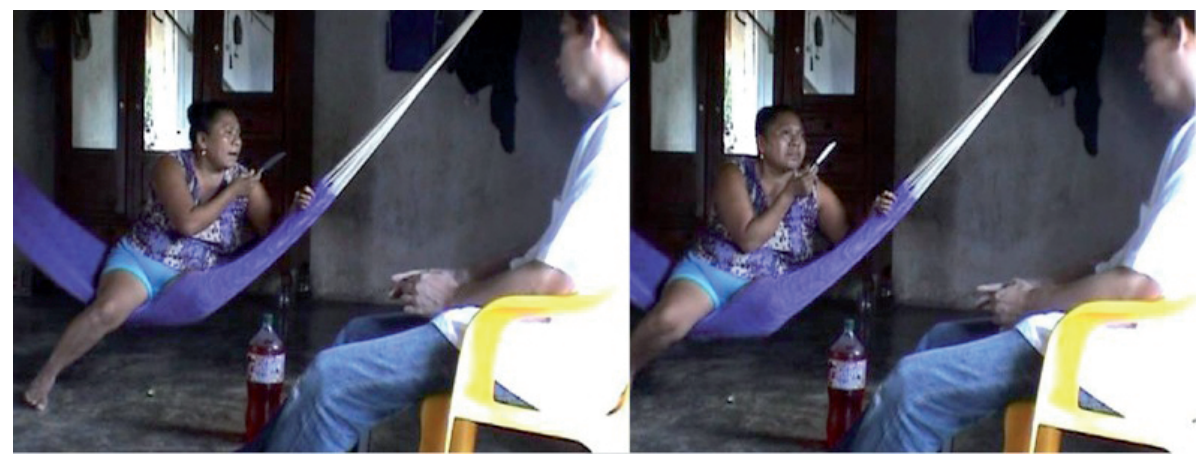

Figure 3. 'well, when (the Saint) leaves (a) [(from) here]'; (b) '(it's) [Hazil]'

Even when the same speaker uses pointing anaphorically, i.e., a point used to refer back to a previously mentioned target without explicit reference to it in speech, ${ }^{4}$ the pointing is still subject to the condition of truth regarding accurate orientation when it comes to communicating existing entities or places. In example 2, W. continues her description of the Saint's journey and now mentions another village called Yo' Aktun, located west of the village (remember W. is facing east). In the first mention of the name of place, W. does not point, but she explains that people in this village go through the same ritual processes she explained earlier. At the end of her utterance, W. points anaphorically: she points to the village but without mentioning its name. The reference is done with the gesture stroke co-occurring with the words "like too" (meaning something like "there in Yo' Aktun too") and the distal enclitic $-o$ ' (Figure 4).

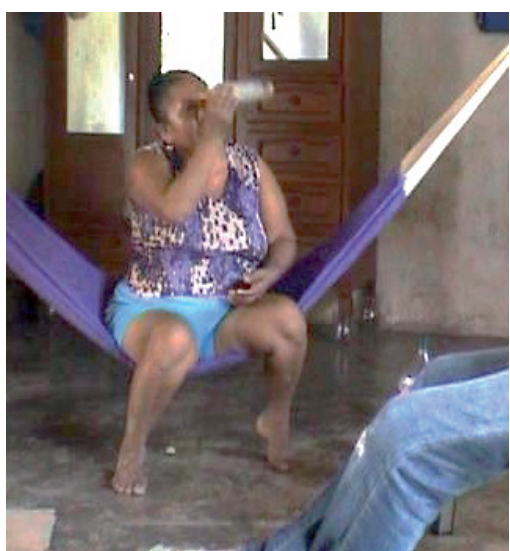

Figure 4. 'It is always the same happening, they say, the food (offering), the bullfight, everything, [like (there in Yo' Aktun) too]' 


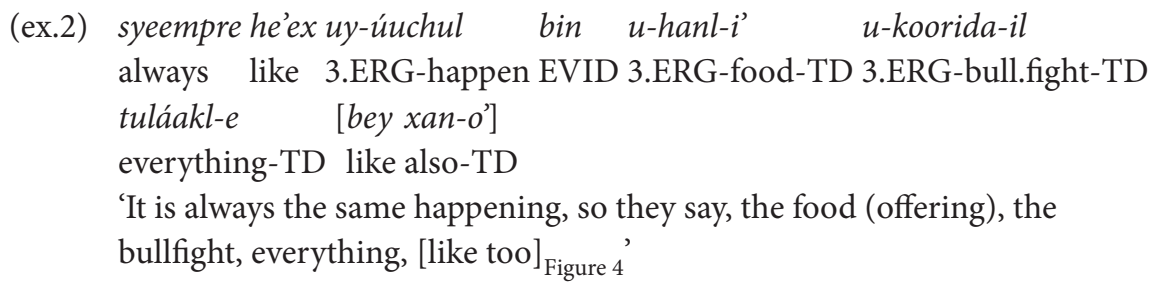

In using a pointing gesture as a discursive device to refer to the village she just mentioned, W. is performing a 'composite utterance' distributing information between her speech and her gesture (Enfield, 2009; Kendon, 2004). The intended meaning of this composite utterance is to be understood by the listener as follows:

Speech It is always the same happening, so they say, the food (offering), the bullfight, everything [like too]

Gesture [pointing towards Yo' Aktun]

Comp. utt. 'It is always the same happening, so they say, the food (offering), the bullfight, everything like [it is the case there in Yo' Aktun] too'

\section{Metonymic pointing}

In metonymic pointing (called 'deferred' in Borg, 2002, or Quine, 1968) the body is the origo and the extended arm or finger represents the vector. The target is a representation of the intended target is metonymical (another entity in relation of contiguity with the target that stands for it) or synecdochal (a component of the target is used to refer to it). A typical example of metonymic pointing is pointing to an empty chair someone has just left in order to refer to this person. In such a case, the link between the chair and the person is provided by the context (unless only this person ever sits in this particular chair). Some entities occupy a stable position in space that can stand for them, such as the sun, especially in the tropics where it has a stable journey. The usual place of the sun in absentia can be used with metonymic pointing to indicate the time of the day. Example 3 presents a case of pointing at the position of the sun at night by a Yucatec Maya speaker. In this example, the speaker is telling a story that happened to him around $10 \mathrm{am}$. To indicate the time, he points to the position of the sun in the east where the sun habitually stands at this time in the morning (Figure 5).

(ex. 3) ha' yàan kex las dyèes k'iin [bey-a']

INTJ EXIST even DET ten sun/day MAN-TD 'yes, it was like 10 AM [like this $]_{\text {Figure } 5}$ '

Although, metonymic pointing to the sun shares a truth condition with direct pointing, the target has a metonymical feature (the position of the sun stands for 


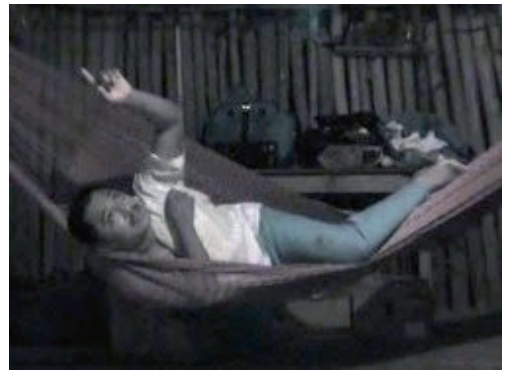

Figure 5. 'yes, it was like 10 AM [like this]'

the sun and therefore the time of the day). Note that more complex metonymic pointing exists, also with reference to the sun (Floyd, submitted) or to the moon (Le Guen \& Pool Balam, submitted).

\section{Metaphorical pointing}

In metaphorical pointing (also called deixis at phantasma by Bühler (1983) or $a b$ stract pointing by McNeill (1992, p. 173)), the target is a region of the space surrounding the speaker used as a discursive reference (Bühler, 1983; Fricke, 2002; McNeill, Cassell, \& Levy, 1993). Because the speaker points at empty space to refer to an actual place, the relationship between this place and the referent is arbitrary. ${ }^{5}$ Consequently, speakers can use this type of pointing to metaphorically refer to real but also distant or unknown places (i.e., the accurate orientation of the place is unknown) as well as abstract concepts. ${ }^{6}$

The origo of the pointing is by default the body of the speaker but it can be reduced to the wrist or to the first phalange of the index finger. The orientation of the vector is relevant above all when it is contrastive with a previous metaphorical pointing. Imagine a speaker saying that he went home and then to his work place and performing two points: the first to the right with the thumb and the second to the left with the index finger (Figure 6). The anchor point of the pointing is not accurate with respect to the orientation of the actual locations of these places. The idea of such a pointing is to oppose maximally the home and the office to discursively emphazes the distance or/and the time it takes to travel between these two places. Figure 6 is an illustration of a metaphorical pointing but examples from naturally-occurring speech can be found in McNeill (1992, pp. 174-175).

In the data analyzed from Yucatec Maya (almost an hour of spontaneous data in four different contexts with 4 different speakers, 3 men and 1 woman), no metaphorical pointing was found in reference to existing space or entities. Because of the condition of truth regarding pointing to actual targets ("pointing should be accurate in terms of angular direction"), such pointing is rare or absent. ${ }^{7}$ On 


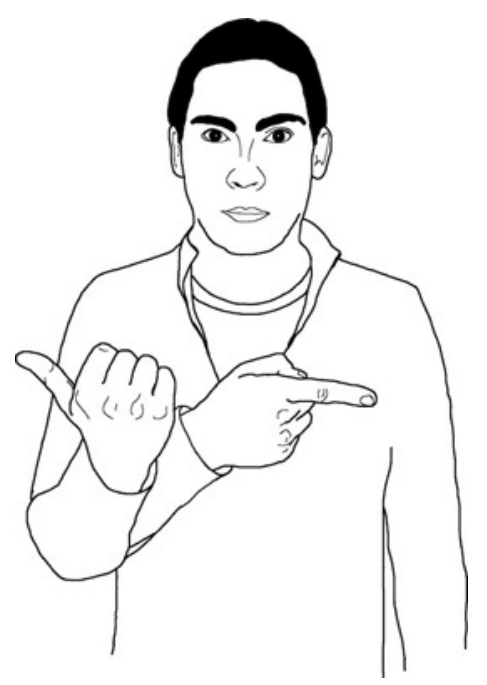

Figure 6. 'I first went home [thumb pointing] before going back to the office [index pointing]'

the other hand, among speakers of English, Dutch or Japanese (mainly egocentric coders, see Levinson, 2003, p.228), metaphorical pointing is considered acceptable even when dealing with existing places or entities. In fact, a low degree of accuracy in pointing to distant places is expected and Schegloff (1984) remarks that in natural interaction, North American English speakers do not show any consistency at this level of information relevance. North American English speakers can use space in a metaphoric way and sometimes point to refer to entities in space without considering their real position. What triggers the use of metaphorical pointing is 'psychological distance' (Trope \& Liberman, 2010), sometimes, but not always, correlated to actual distance and/or memory. Schegloff notes that: "If the place referred to is not visually accessible, then it appears that the point is not necessarily in a direction selected to be the "actual direction" of the referent relative to the scene of the talk. (...) Different "places" (which happen to be in different directions from the talk scene) are accompanied by points in the same direction, and two persons referring to the same place while talking together point in different directions" (Schegloff, 1984, p. 280). Since egocentric speakers can point to actual space metaphorically, they do not need interactionally to constantly compute their position because their pointings are not subject to a condition of truth (i.e., orientation of the pointing does not have to be accurate). This communicative tendency may explain in part their poor performance in pointing tasks (to distant places) relative to geocentric coders (see Levinson, 2003, chap. 6).

To try to sustain the claim that Yucatec Maya are reluctant to use metaphorical pointing (and because it is always difficult to prove that something does not exist, 
because there is no data(!)) consider this example of metonymic pointings extracted from a narrative that happened in a virtual place (I am reluctant to use the term fantasy because Yucatec speakers do believe it happens, it just that nobody knows where and when exactly). In this narrative, since the speaker does not know where the events happened, he is mapping the elements of the story on the current speech situation. For instance, when he talks about the garden of the protagonist he points to the garden in the house where he currently stands. At some point in the story, the man pretends to go to his field and goes instead to see a ritual specialist (or $h$ meen). The speaker performs two pointings (done in a single arc movement but with pauses) indicating two places: the field of the protagonist and the place of a ritual specialist. Interestingly, the first pointing is done towards the west (Figure 7a), where the majority of villagers' fields are located (and in particular the one of the speaker). The second pointing is done to his back, towards north (Figure $7 \mathrm{~b}$ ). In his utterance, the speaker mentions that the protagonist went to see the "great man" (a term equivalent to $h$ meen).

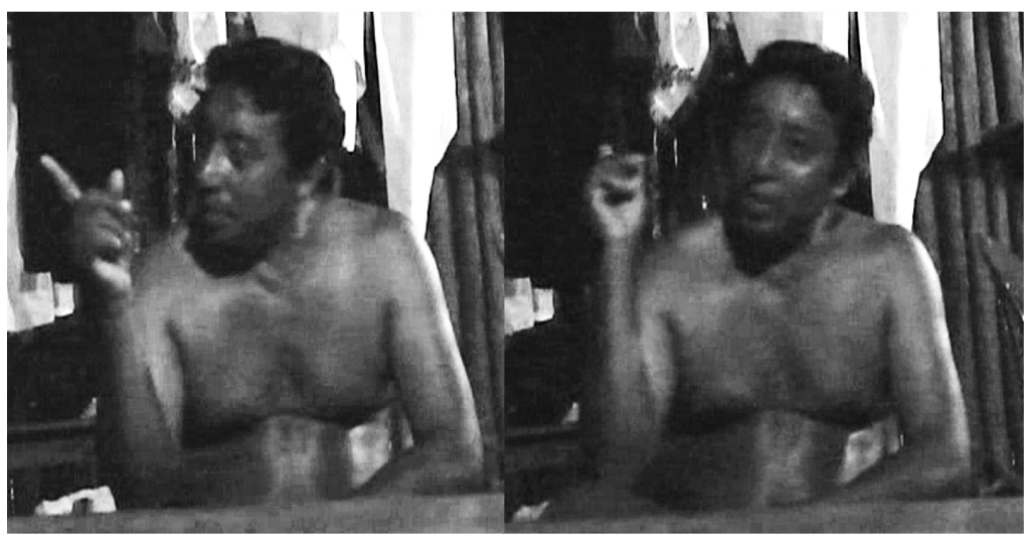

Figure 7. '[but he did not go to (his) field], [instead he circled it] and went to see the great man'

(ex. 4) [pero ma' ich kool bin-i' sino] $]_{\text {Figure 7a }}$

CONJ NEG in field GO-TD CONJ

[ke bin $t$-u-meet-ah $u$-ba'paacht-e'] $]_{\text {Figure } 7 \mathrm{~b}}$

CONJ GO CP-3A-DO-CP-TR 3A-go.around-TD

ka' h-bin yikn-e nohoch máak-o'

CONJ CP GO at-TD great man-TD

'but he did not go to (his) field, instead he circled it and went to see the great man (=ritual specialist)'

At first glance, the second pointing (Figure 7b) looks like a metaphorical pointing indicating a piece of empty air behind the speaker, metaphorically representing a 
remote or unknown place. This is not the case. The speaker intentionally points to this direction and indicates a village to the north (San Andres) where the ritual specialist the people from Kopchen usually goes consult. ${ }^{8}$ The two pointings can be considered metonymical because the speaker points to existing places to refer to places in the narrative (the field and the house of the ritual specialist of the narrative). But more importantly, it seems that Yucatec Maya speakers are reluctant to use metaphorical pointing to places, even hypothetical places in narratives, and tend to map them on to real places in their surrounding, using direct or metonymic pointing. This analysis sheds a new light on previous interpretations of Yucatec Maya gestures by Kita et al. (2001) who looked only at the use of the axis (frontback and left-right) without considering the possible mapping to existing places.

\section{Pointing in transposed settings: Using the frames of reference}

When speakers gesture about space, especially about distant space, they need to specify how they encode the relation between a distant figure and a distant ground. In this case, the vector indicated should not be understood as starting from the body but from a transposed origo in a distant or so-called 'laminated space' (Hanks, 1990; Haviland, 1993, 1996). The condition when the Narrated Event (what is described) does not correspond to the Speech Event (where the speech is occurring) (Jakobson, 1971) is called 'transposition.' Transposition occurs when a speaker is talking about a place that is not the space where his or her body is located, that is, when the origo of the vector described is different from the current body position of the speaker. Transposition occurs in two contexts for spatial indications:

1. if the speaker is talking about a figure and a ground located in a distant place

2. when the speaker, in the process of uttering some direction-giving that started from his or her current position, describes the second segment of a path

Transposition is a complex process because, although speakers gesture about a distant space, they have to do it with respect to their own body and their current position in space. In this paper two solutions are considered for talking about distant space using frames of reference: using the egocentric FoR (projecting an imagined point of view of the distant scene being described) or using the geocentric FoR (making use of the real world orientation and actual places of the entities to be located). Crucially, following Levinson (2003), the geocentric and the egocentric FoR impose a ternary relation to determine the position of the figure in relation to the ground. In other words, in the geocentric FoR the body can be the ground (e.g., "north of me") but the anchor point is always extrinsic (e.g., cardinal 
directions). In the egocentric FoR, the body is never the ground and the anchor is always extrinsic determined by the point of view of the speaker. This contrasts with the intrinsic FoR that only involves a binary relation where the anchor point lies within the ground, more precisely it is determined by the internal division of the ground, which can be the body in some cases. However, under transposed conditions the body cannot be the ground. Therefore, the use of the intrinsic FoR under transposition rules out the use of the body as ground. Additionally, the intrinsic FoR is useful only when the ground has an internal division and becomes unavailable when it is not the case (e.g., an intrinsic utterance like "the Figure is in front of the tree" would be impossible because the tree has no front or back). Finally, the intrinsic FoR can be ambiguous in specific cases when the ground has several contiguous fronts or backs for instance (as in the example of the gas station, see below and Le Guen, 2011).

The choice of using the egocentric or the geocentric FoRs is mainly determined by the speakers' cultural habits (although, as discussed below, knowledge of the local geography influences this choice). However, the egocentric and the geocentric FoR make use of different cognitive strategies that have both advantages and constraints.

In direction-giving and sometimes in descriptions of the relative placement of entities, speakers rely on a process of segmentation. Because of the constraints of human communication, speakers have to transform their mental representation into a temporal flow using the structure of the language that imposes a linearization and segmentation of the information. The communication of information is done in the form of verbal propositions and gesture where some cues mark the beginning and sometimes the end of each segment. Segmentation also facilitates understanding and memorization for the interlocutor. In the process of segmentation, every new segment (besides the one that starts from the body of the speaker) will create a new transposed origo. What distinguishes the use of the egocentric and the geocentric FoRs is how the transposed origo is created and what stands for it.

\section{The use of the egocentric FoR in transposed pointing}

This section examines how pointings are produced using the egocentric FoR in (1) path indication and (2) placement of distant entities. 


\section{Path indication}

In order to understand how the egocentric FoR is used in direction giving, consider example 5 where a French speaker in Paris was asked how to get from the Sorbonne, where she was standing, to the Boulevard Saint Germain. In order to give the directions, she decomposed the path into two segments (see example 5 and Figure 8). The first segment starts from the Sorbonne (where the speaker's body is located) and ends at the intersection between the Place de la Sorbonne and the Boulevard Saint Michel. According to the classification proposed above, this point is a direct point where the place of the body (that includes the Sorbonne) constitutes the ground and the extended arm indicates the direction where the interlocutor should go. The intersection could be seen as the figure (the target), but this is not really necessary in path indication since only the direction of the vector is the relevant information. Besides, the segment usually ends at the projection of the next origo (i.e., at the beginning of the next segment). The second segment begins from the intersection in front of the Sorbonne and ends at the intersection with the boulevard Saint Germain (see Figure 10).

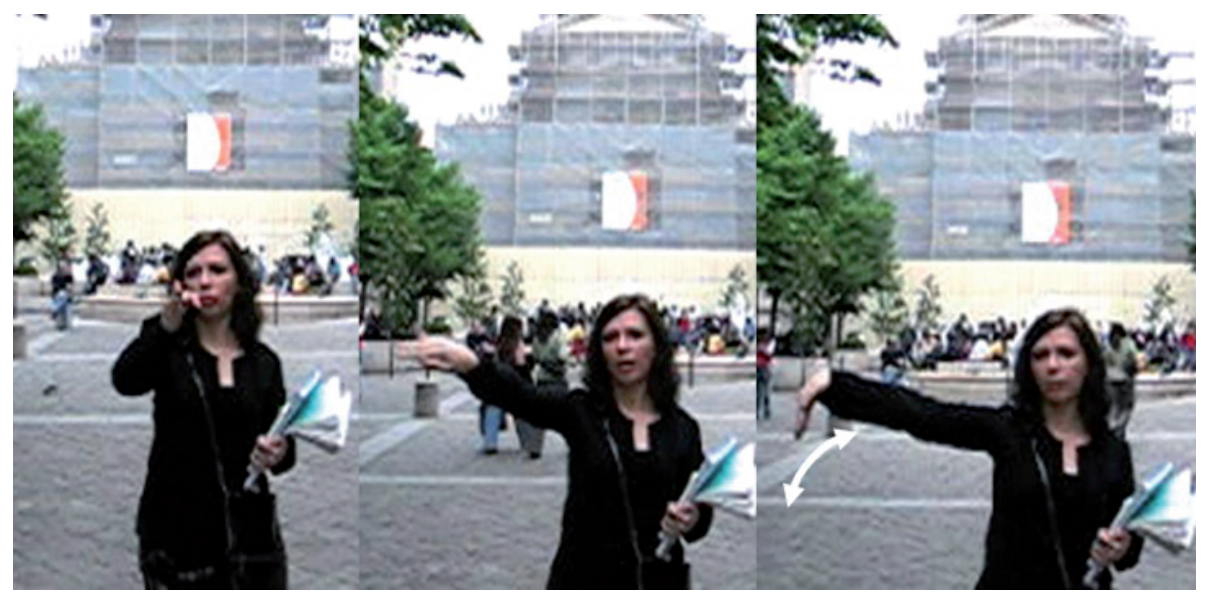

Figure 8. (a) 'I [go straight to the Boulevard Saint Michel]'; (b) '[I go down on my right]'; (c) '[the Boulevard Saint Germain is only straight like this]'

(ex. 5) Je [vais tout droit jusqu'au Boulevard Saint Michel]

'I [go straight to the Boulevard Saint Michel]'

[je descends sur ma droite]

'[I go down on my right]'

... [le Boulevard Saint Germain est juste toujours tout droit comme ça]

'... [the Boulevard Saint Germain is only straight like this]' 
To indicate the first segment, the speaker says "first, I go straight to Boulevard Saint Michel," and extends her arm in front of her accordingly (Figure 8a). This gesture is a direct pointing, her body corresponding with the starting point of the path. When she utters her second indication: "I go down on my right," she extends her arm accordingly to her right (Figure $8 \mathrm{~b}$ ). This indication should not to be taken as starting from the current position of her body. The speaker does not intend to say 'it's to the right side of my body.' Instead, she assumes that the interlocutor projects himself to this transposed origo, the intersection, and what she intends to say is: '[at the intersection], make a right turn.' In her second utterance, the Origo of the Speech Event does not coincide with the Origo of the Narrated Event (i.e.: $\left.\mathrm{O}_{\mathrm{ES}} \neq \mathrm{O}_{\mathrm{EN}}\right)$. The $\mathrm{O}_{\mathrm{ES}}$ is the speaker's body at the Sorbonne, whereas the $\mathrm{O}_{\mathrm{EN}}$ is the intersection. The last gesture (Figure $8 \mathrm{c}$ ) is a hand sweep to indicate the internal orientation of the Boulevard Saint Germain.

When the path does not start from the place where the speaker is currently speaking, the origo of the narrated event differs from the origo of the speech event (as in the indication of the second segment in example 5). However, the more distant the projected origo is from the origo of the speech event, the more dramatic the differences in gesture production become and the more the frame of reference used should be made clear. In the case of the egocentric FoR, some cue regarding the view point of the speaker of the projected scene are expected.

In example 6 and Figure 9, the same speaker was asked to indicate the same path, but this time from a different location (the Sénat, also in Paris, see Figure 10) and from a different orientation, she is now rotated 180 degrees from her first position (at the Sorbonne).

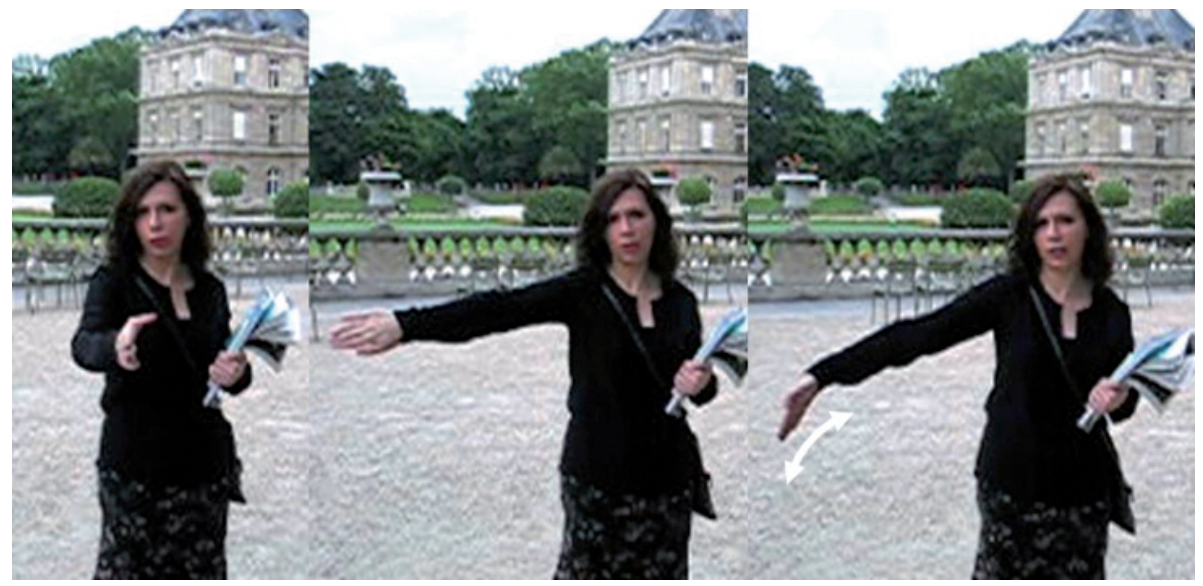

Figure 9. (a) 'I go [straight and meet the Boulevard Saint Michel]'; (b) 'and [I go down, to the right]'; (c) 'and I encounter [the Boulevard Saint Germain]' 
(ex. 6) si la Sorbonne est derrière moi,

'if the Sorbonne is at behind me

je vais [tout droit, je rejoins l'Boulevard Saint Michel]

'I go [straight and meet the Boulevard Saint Michel]'

et [j'descends à droite]

'and [I go down, to the right]'

et j'tombe [sur le Boulevard Saint Germain]

'and I encounter [to the Boulevard Saint Germain]'

In contrast with her first position, the origin of the path indicated by the speaker is not her current body position: she is at the Sénat and the path starts from the Sorbonne. That is, the origo of the Speech Event does not coincide with the one of the Narrated Event $\left(\mathrm{O}_{\mathrm{ES}} \neq \mathrm{O}_{\mathrm{EN}}\right)$. A rapid comparison of the verbal indication of the speaker in the first position (example 5) and in the second position (example 6) shows that they are similar, except with one detail: the explicit mention of the Sorbonne being behind her back in the second position. The mention of the Sorbonne has two effects: (1) it specifies the transposed origo $\left(\mathrm{O}_{\mathrm{EN}}\right)$ from which the path indication will start and (2) it makes clear the orientation of the speaker in the transposed origo (i.e., looking away from the Sorbonne, towards the boulevard Saint Michel). The orientation of the speaker in the transposed origo is crucial, especially to correctly understand the second indication 'to the right.' In other words, when going away from the Sorbonne, one would have to rely on one's body orientation (more precisely one's view point) to make a correct turn to the right.

A comparison of the speaker's gesture production in both positions reveals that they are similar and that they echo the verbal indications. But crucially, gestures performed in position 2 have to be understood from the point of view of the speaker in the transposed origo (i.e., a right hand pointing means a right turn looking away from the Sorbonne). Figure 10 indicates the two positions of the speaker on a map of Paris with the two segments: A-B (the Sorbonne to the intersection) and B-C (the intersection to the Boulevard Saint Germain). The gestures of the speaker are identical (using the front-back axis and then the left-right axis). In both positions, angular information is provided from the point of view of the speaker: the current point of view in position 1 and the projected point of view in position 2.

From Figure 10, we can notice that only in position 1 are the speaker's gestures performed in accordance with the real orientation of the path. In position 2, the gestures of the speaker are mirrored and therefore inversely oriented in comparison with position 1: first she points to the east and then to the south whereas the segment $\mathrm{AB}$ is oriented west from the Sorbonne and the segment B-C north of the intersection. 

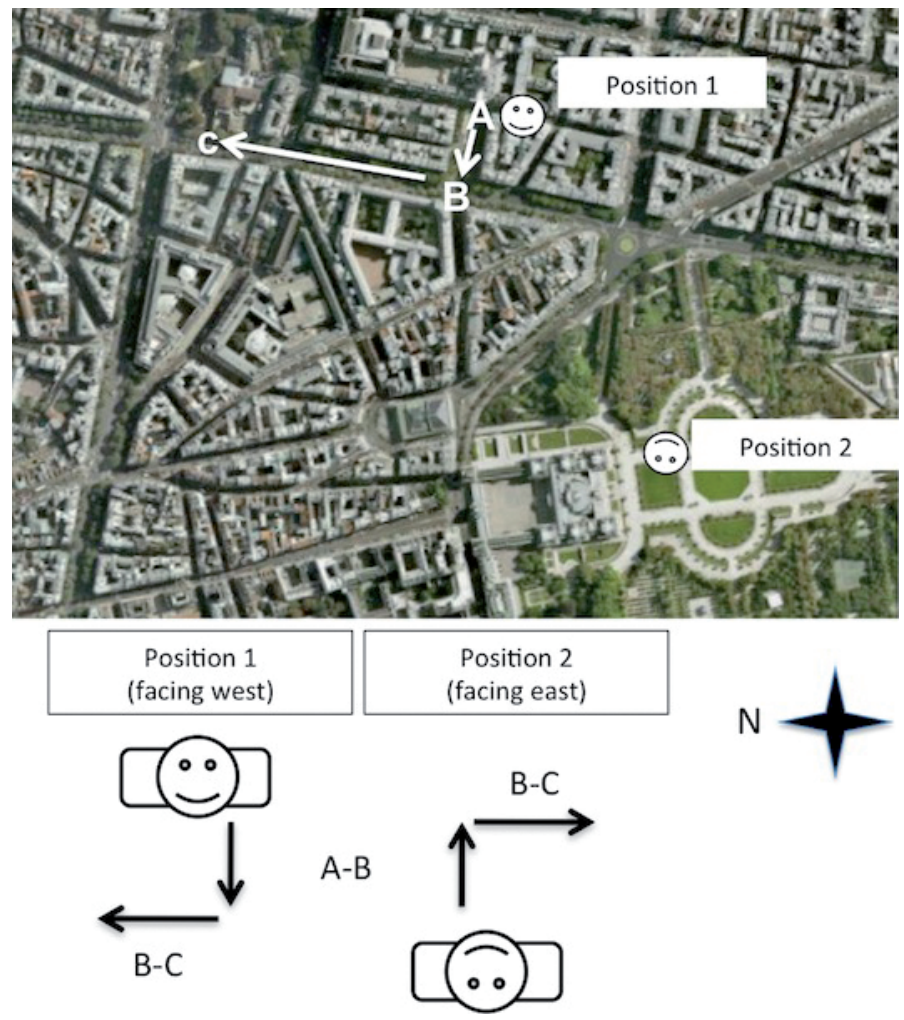

Figure 10. Comparison of the French speaker's gestures (the black arrows indicate the orientation of the speaker's gesture)

\section{Placement of entities in distant space}

Using the egocentric FoR to place distant entities relies on the same strategy as direction-giving, that is, the projection of a point of view. In the case of placement of distant entities, the origo projected is not the beginning of a segment but a point of view on a scene that determines the search domain from the ground to locate a figure.

Imagine a speaker A explaining to his interlocutor B how to get to his house. Imagine A saying that his house is the one to the left of a tree when coming from a specific direction. As he utters his indication, A also accordingly performs a pointing to his left (Figure 11). It does not matter what direction $\mathrm{A}$ is facing at the moment of uttering his indication in the speech event because $\mathrm{A}$ is projecting an imagined point of view on the scene (i.e., in the narrated event) in order to attribute a left side to the tree (the ground). Specifying point of view in the narrated event (e.g., using verbal cues such as "coming from $x$ " or "looking at $x$ ") becomes critical when the ground has no orientation, as in the case of a tree for instance 


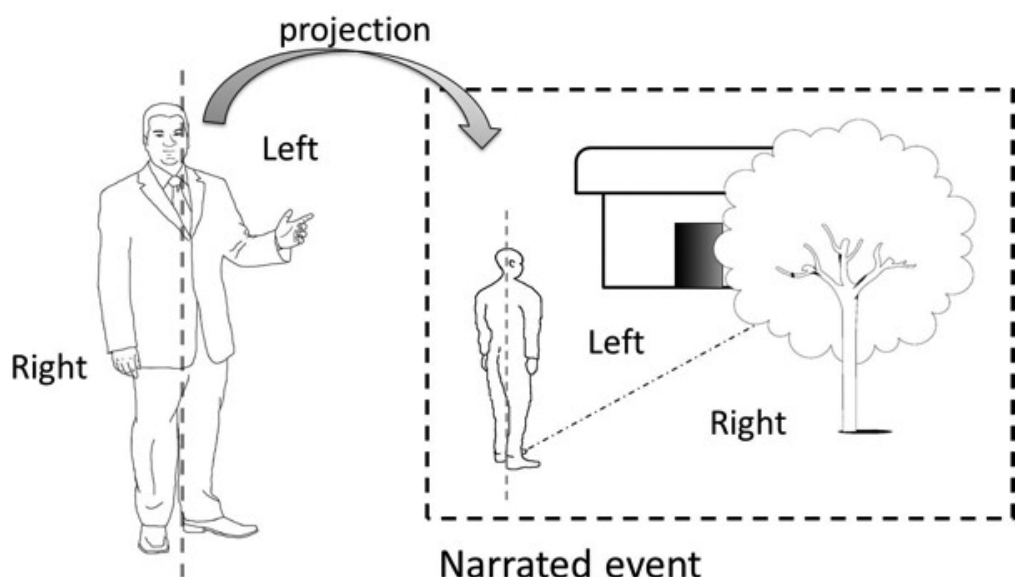

\section{Speech Event}

Figure 11. 'the house is to the left of the tree'

(note that the intrinsic FoR would not be sufficient to specify the spatial relationships in this case). As for direction giving with the egocentric FoR, we notice that the gesture production echoes the verbal indication: the person says to the left and points to the left.

\section{The use of the geocentric FoR in transposed pointing}

In contrast with the use of the egocentric FoR, no point of view is involved in the geocentric FoR. Geocentric coders rely on some extrinsic features of the environment to define spatial relationships. Since speakers assume the shared knowledge of the environment and the stability of the entities to be located in space, they can afford to be more elliptical in their verbal indications, as it is the case with Yucatec Maya speakers.

\section{Path indication}

Elicitation task. Two Yucatec Maya speakers were asked to indicate a distant path comparable to the one presented in the French examples (ex. 7 and 8). Both participants were asked to indicate a path that does not start from their current position (i.e., the origo of the speech event differs from the origo of the narrated event: $\mathrm{O}_{\mathrm{ES}} \neq \mathrm{O}_{\mathrm{EN}}$ ). The path they have to indicate starts from the church of the village and ends at the house of Don Y (at the other end of the village). This path was segmented by the speakers into three sections as indicated in Figure 14 and goes as 
follows: A-B (church to the first intersection), B-C (intersection 1 to intersection 2) and C-D (intersection 2 to Don Y's house). Crucially, both were asked to indicate the same path, but when facing in different directions: an adult male (I.) was interviewed facing south, and a 10 year old girl (M.) was interviewed facing east. In both cases, the camera faces north. Example 7 and Figure 12 presents the adult male verbal and gestural indication and example 8 and Figure 13 the young girl's.

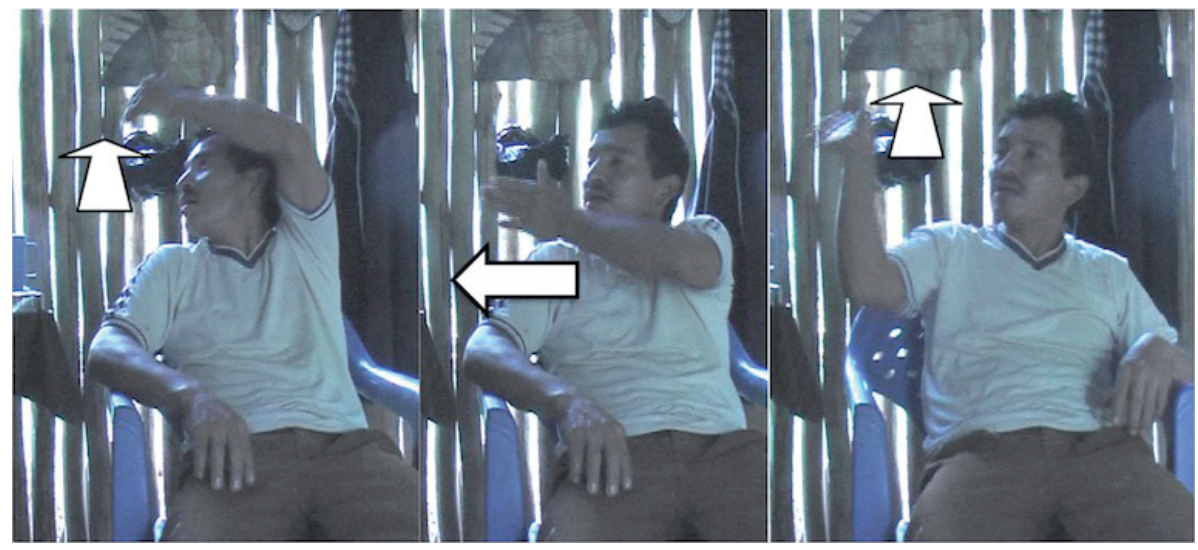

Figure 12. (a) 'You take the [street like this straight]' ... (b) '[you take like this]', ... then, (c) '[you take again there like this]'

(ex.7) $k$-a-ch'a'-ik e [kaaye'bey toh-a']

AM-2E-take-TR.IC DET street MAN straight-TD

ken k'uch-k-ech t-u-eskina t-u-nayl-e $P$.

AM arrive-SBJ-2A FOC-3E-corner FOC-3E-house-TD name

[k-a-ch'a'ik bey-a'] t-u-'eeskina u-nayl C.

AM-2E-take-TR.IC MAN-TD FOC-3E-corner 3E-house name

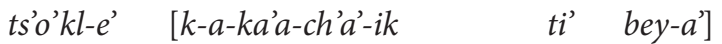

TERM-TD AM-2E-again-take-TR.IC FOC MAN-TD

'You take the [street like this straight]. When you get to the corner of P's house, [you take like this], at the corner of C's house, then, [you take again there like this]'

(ex.8) Ken xi'ik-en [bey-a'] [k-in-bin bey-a']

AM go.SBJ-1E MAN-TD AM-1E-go MAN-TD

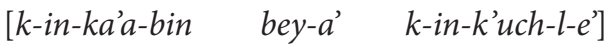

AM-1E-again-go MNA-TD AM-1E-arrive-NOM-TD

'I [would go like this], (and) [I go like this], (and) [I go again like this (and) I arrive]' 


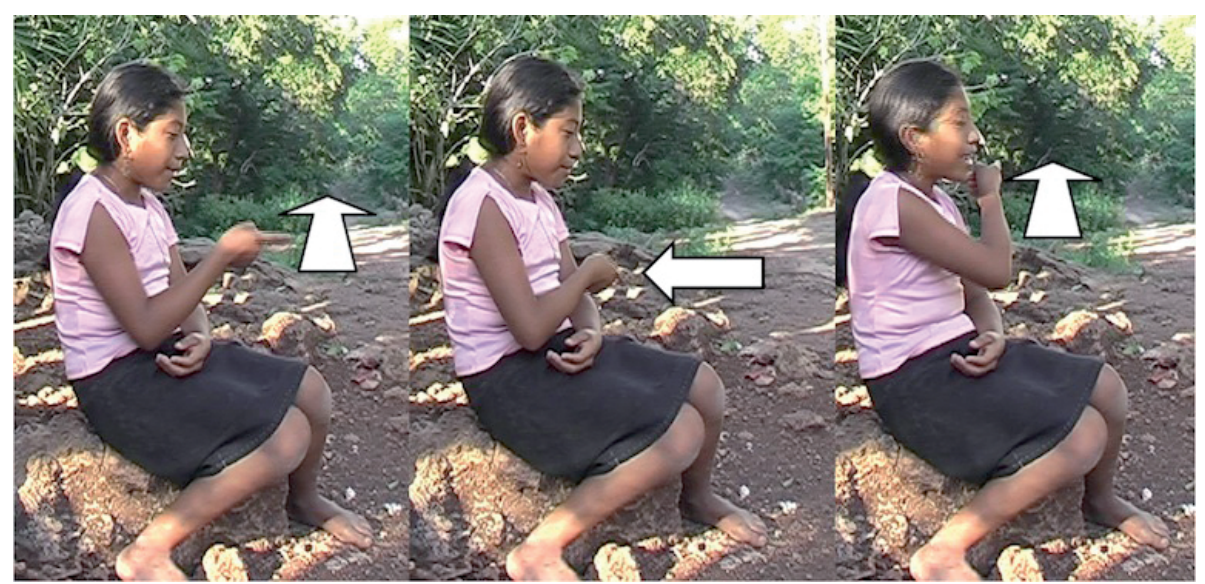

Figure 13. I (a) [would go like this], (b) [I go like this], (and) (c) [I go again like this]

In comparison with the French examples, we immediately notice that (1) verbal instructions are not sufficient to understand the direction (i.e., the angular information) and (2) the gestures of the two speakers are produced differently.

Verbal indications are quite elliptic. Neither speaker gives any cue regarding what FoR they are using: they do not mention spatial terms such as left or right or cardinal directions. Instead, they use manner deictics ('like this'). The use of deictics has an important consequence: directing the attention of the listener towards the gesture. Looking at both speakers' gestural production, we notice that they are not similar according to their body axis but rather are produced according to the actual orientation of the segments of the path from each projected origos (see Figure 14). Because the speakers face in different directions, I. indicates the first segment with a gesture oriented south-north using the front-back axis of his body (Figure 12a). In contrast, M.s gesture is performed along the left-right axis (Figure 13a), encoding the same south-north orientation. For the second segment, I. now uses the left-right axis of his body to encode the east-west orientation of the segment (remember he is facing south, Figure 12b) while M. uses the awaytowards the body's axis (Figure 13b). Finally, the last segment is performed like the first (note both speakers' use of ka'a 'again' in specifying the third segment). The transposed origo, as with the French examples, is always the beginning of the next segment. However, in contrast with the French examples, the landmark, in this case the street-corner, is the transposed origo and not the place where the speaker imagines himself looking at the scene. 


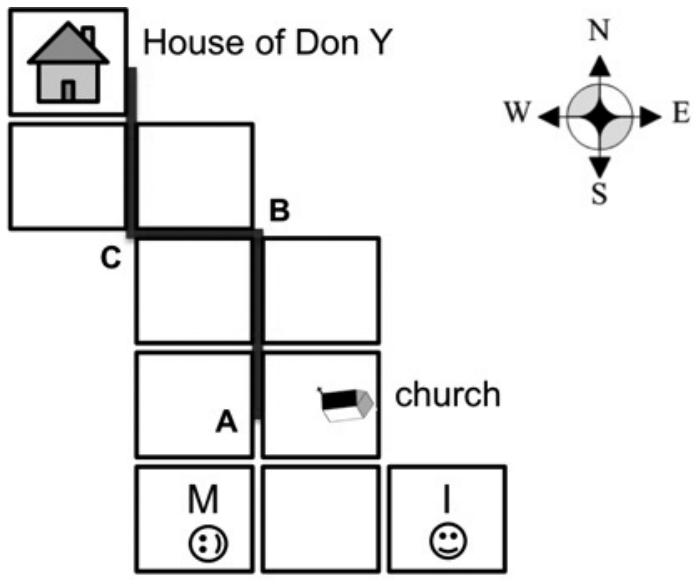

\section{M (facing east)}

\section{I (facing south)}
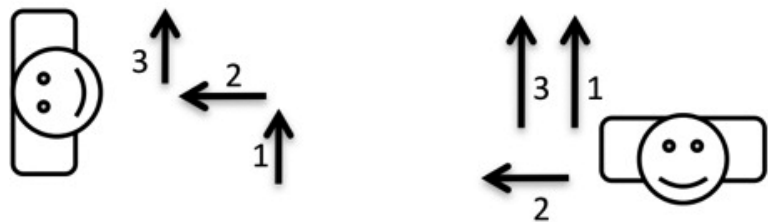

Figure 14. Comparison of I. and M. gestural indication with respect to the orientation of the path (the black arrows indicate the orientation of the speaker's gesture and the numbers the segments indicated)

\section{Communicating placement of entities in distant space}

Under transposition, in contrast with egocentric coders who need an imagined viewpoint in the narrated event, geocentric coders recreate the relation between a figure and the ground using the real world orientation (e.g., in terms of cardinal directions).

In the following examples of elicited data, twenty Yucatec Maya participants were asked to recreate a distant array composed of a shop (the figure) and a gas station (the ground) in the town of Felipe Carrillo Puerto situated $30 \mathrm{~km}$ north of the village. The shop is called Azulero (AZ) and is located in front of the Gas station (GS) on the north side (see Figure 15). Cars can enter on both the east and the north sides of the gas station, so it has no single intrinsic front or back. All participants were familiar with the arrangement.

The question asked to the participants was phrased as follows: "In Felipe Carrillo Puerto, where is the Azulero in relation to the Gas Station?" (Te' kàariyoo', 


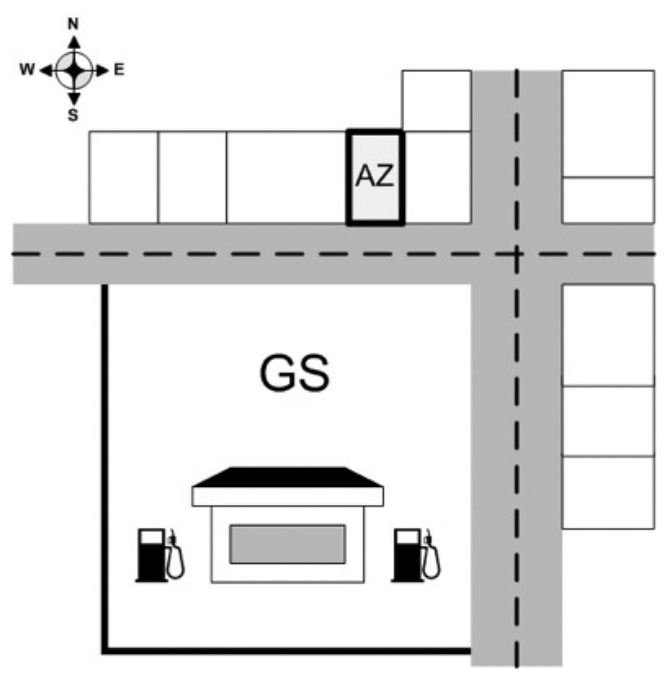

Figure 15. The Azulero-Gas station arrangement (eye bird view)

tu'ux yàan le àasuleroo' te' Gasolinerao'?). Participants were free to give any kind of response they wanted to as long as their responses contained an explicit mention of the figure and the ground (that is, where the shop was located in relation to the gas station). Participants were divided into two groups, in the first participants (5 women and 6 men) faced west and in the second ( 4 women and 5 men) they faced south (more detail about this task can be found in Le Guen, 2011).

Results show that participants in both groups used a geocentric FoR in their gesture production: they all placed the figure (the AZ) north of the ground (the GS). As with previous examples of path indication (ex. 7 and 8), verbal indications are elliptic (only 2 men used the word xaman, 'north') but we notice a consistent use of manner deictics. Speaker's verbal indications consist mainly in composite utterances such as 'the AZ is on the side of the GS [with a point to the north]' or 'the AZ is like this [pointing to the north] and the GS is like this [pointing to the south].' The under-specification in the linguistic channel is accounted for by the fact that the relevant angular information is provided by the gesture to which attention is triggered by the deictic expression in the speech (note that it is also the case in the French examples but only to iconically represent how the boulevard is encountered).

As in examples 7 and 8, gesture production of the speakers differs (i.e., with respect to their own body) according to their orientation, but it is always produced in accordance with the actual north-south orientation of the AZ-GS array. Participants that faced west used the left-right axis of the body, placing the AZ to their right (i.e., the north side of their body) and the GS to their left (i.e., the south side of their body), see Figure 16. Accordingly, participants that were facing south 
placed the AZ to the north side of their body (or northward) and the GS to the south side of their body (or southward), see Figure 17. While 8 of 9 participants in group 2 pointed behind them to place the AZ and in front of them to place the GS, one participant used his body as the AZ and placed the GS away from his body in front of him, i.e., southward (Figure 17c).

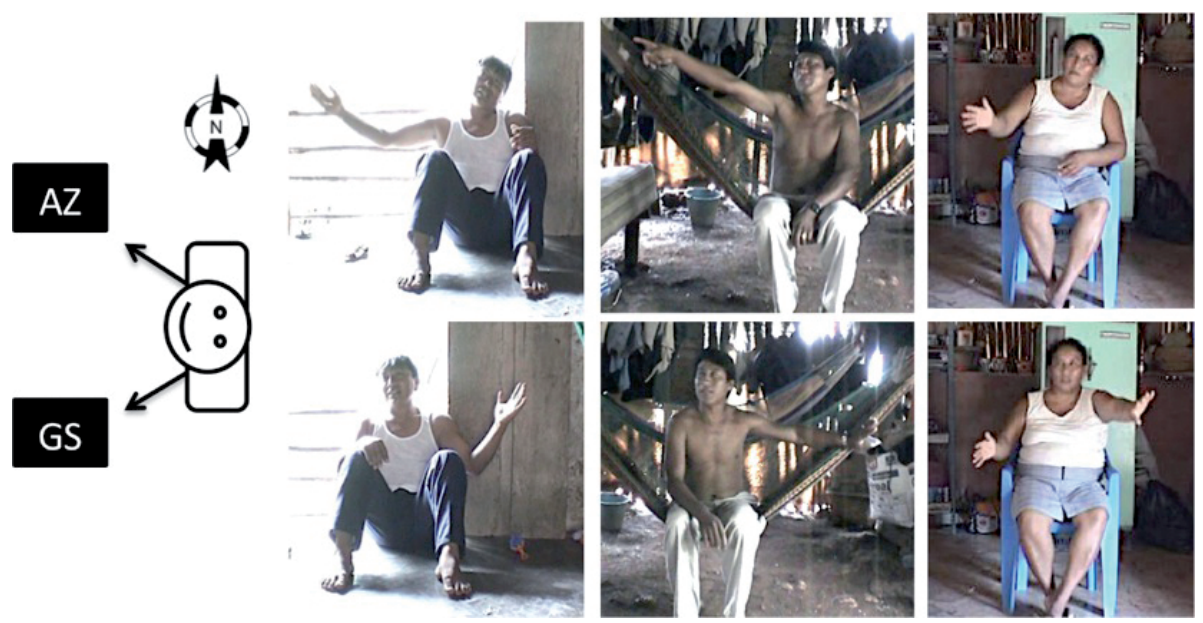

Figure 16. The $\mathrm{AZ}$ is placed to the right side (to the north) of the speakers' body (row above), and the GS to the left side (to the south) of the speakers' body (row below).

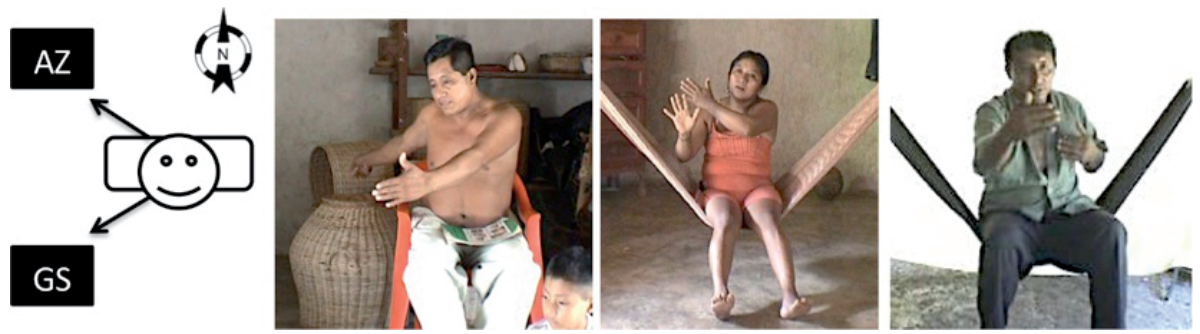

Figure 17. The AZ is placed to the north or northward of the speaker's body while the GS is placed to the south or southward of the speaker's body

Interestingly, although the participants point or place their hand in empty air, they do not use metaphorical pointing because, (1) the relevant origo is not their body but the transposed origo and (2) they recreate the spatial relationship between a distant figure and a distant ground taking into account their real-world relationship (in this case north-south). Most of the speakers used their body axis to oppose the position of the AZ with respect to the GS.

In contrast with path indication and placement of distant entities done with the egocentric FoR, the position of the transposed origo has to be inferred. In the AZ-GS array, only the figure-ground relation is clearly established by the speakers. 
This contrasts with the use of the egocentric FoR where the point of view and the speaker's orientation in the narrated event is made clear.

Since point of view is irrelevant while using the geocentric FoR, no cues regarding the speakers' orientation or point of view in the transposed origos are given, nor are they necessary for an optimal transmission of information. No matter what the orientation of the speaker is in the speech event, the orientation of the stretched arm (i.e., the vector) is always congruent with the real world orientation of the referents. In other words, the orientation of the vector from the transposed origo (or the ground) is the same as the orientation from the body. To illustrate this point, imagine a similar scene as presented above where $\mathrm{A}$ is telling $\mathrm{B}$ where to find his house. While an egocentric speaker would use a process of projection (see Figure 11), a geocentric coder will rely on the real world orientation and use a process of translation, as schematized in Figure 18. Note that there is no discrepancy between the orientation of the speaker in the speech event and the narrated event. In this example the landmark (the tree) is used as the projected origo.

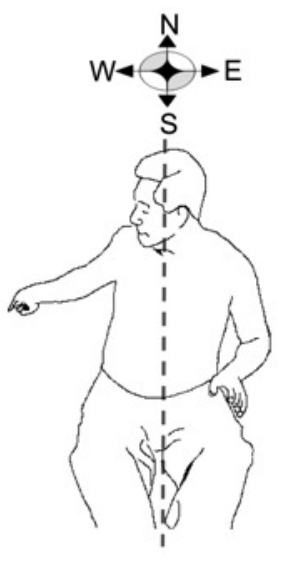

Speech Event

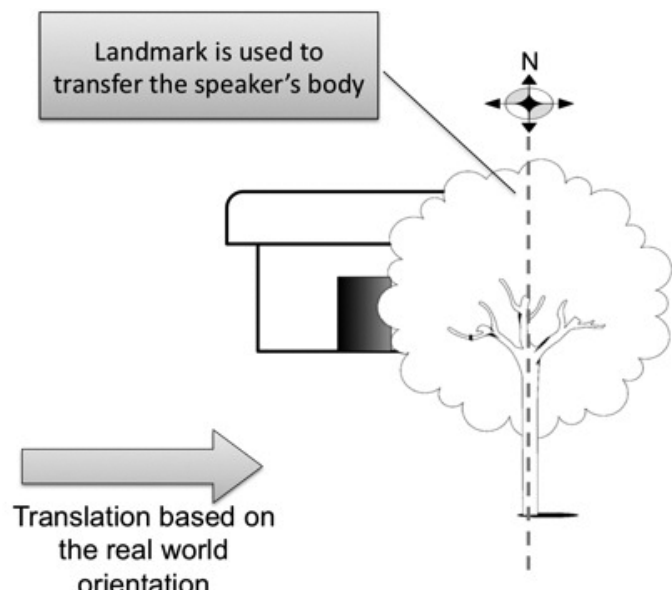

Narrated event

Figure 18. 'the house is to the north/on this side of the tree'

\section{Discussion}

In non-transposed pointing, i.e., where the Speech Event is equal to the Narrated Event, the body of the speaker is always the origo (i.e., the figure). What needs to be defined is the status of the target, giving rise to the various types of pointing: direct, metonymic and metaphorical (see Section 1). 
Under transposition, i.e., where the Speech Event differs from the Narrated Event, the relation between the ground and the figure has to be specified. Using the egocentric FoR, a speaker needs to specify an imagined point of view and an orientation in the transposed scene. The speaker's current orientation is not necessarily the one he will be using in the narrated event, therefore the orientation of his gesture should be understood in accordance with the projected point of view in the Narrated Event for path indication as well as for placement of distant objects. On the other hand, speakers using a geocentric FoR do not have to specify their point of view in the Narrated Event because they make use of the real world orientation to define orientation (in path indication) and the relationship between figure and ground (in placement of distant entities).

The habitual use of FoRs has a direct impact in the types of pointings used in a speech community. According to the definition proposed for direct pointing, regardless of whether the target is directly visible or if it is spatially remote by thousands of kilometers, the pointing should be performed in the correct angular orientation. Importantly, a point to very distant targets cannot be considered a 'geocentric pointing.' No formal criteria distinguish pointing to the cup on the table from pointing to a distant mountain. In both cases, the origos are the body of the speaker, in both cases the arm is oriented accurately towards the target and both targets are (or should be) cognitively available to the speaker and the interlocutor. What distinguishes egocentric and geocentric coders is the shared knowledge of speakers regarding distant targets and whether or not they are (communicatively) authorized to point to remote entities inaccurately (in terms of angular information) (Haviland, 2000). Additionally, the same rule regarding direct pointing among the geocentric coders seems to reduce the use of metaphorical pointing to existing places. Importantly, these differences reside in the communicative expectations and cultural conventions of a speech community.

\section{The grammaticalization of pointing}

In non-transposed pointing (with the exception of some cases of anaphoric pointing), speech makes obvious the reference to the creation of a vector towards the target in the gestural modality. Very often a deictic or a shifter is used to direct the interlocutor's attention to the gesture produced (the point). In transposed pointings, more information is necessary for the interlocutor to understand the relation of the distant figure to the distant ground depending on the FoR used, egocentric or geocentric. In terms of content, it seems that in the use of gesture with the egocentric FoR, there is semantic redundancy: for instance, when the utterance 'go to the left' is accompanied with a left pointing. This redundancy might servers as an insurance against the obscuring of the message because of noise in the channels. 
Since the orientation of the pointing for egocentric coders under transposition is not always the same as in the speech event, some clarification is necessary. It is not always obvious (without verbal cues) that a left point performed by the speaker means left from the current body position (in non-transposed condition) or to the left from the projected point of view (under transposition).

In the use of the geocentric FoR, there can be redundancy or complementarity: i.e., if speakers can use both the term of a cardinal direction and point at it, they can also spare the verbal information and use a deictic instead that directs the listener's attention to the gesture that carries the relevant angular orientation. Indeed, for geocentric coders, a point to the north of the body always means 'north', in non-transposed condition (north from the body's current position) as well as under transposition (north from the projected origo). Note that among Yucatec Maya, in contrast with spatial deictics ('there') mainly used in non-transposed pointing, manner deictic ('like this/that') are pervasive in transposed pointings for it forced the interlocutor to look at the gesture and not only infer to position of the target from speech only.

If the egocentric system can appear more complicated in terms of communication requirements, it is also more portable. Using the geocentric FoR means that one must constantly keep track of the real world orientation of places and things and one must have some knowledge of the local geography.

\section{Frame of reference shift}

Egocentric coders and geocentric coders, when producing gestural direction giving that begins from their body or a non-remote place, start using the same system. The first segment, as shown above, is a direct pointing. Then, depending on the knowledge of the local geography, egocentric coders can go on producing gestural indication aligned with the actual orientation of the path, this either direct pointing (see example 5 for instance) or the geocentric FoR, although they often have to modify their body orientation accordingly (see Kita, 2003a). When the (psychological) distance, the availability of visual cues, or the knowledge of the geography becomes too remote, egocentric coders shift to a complete egocentric system where the projected point of view does not have to be consistent with the local geography, as in example 6. This shift between FoRs has been noted in Kita (1998) among Japanese speakers. In contrast with egocentric coders, geocentric coders never shift from the geocentric FoR. 
The morphology of gesture and the use of body gestural space

In the literature, the comparison of pointing gesture morphology between egocentric and geocentric coders suffers one main limitation: the failure to distinguish between pointing in non-transposed and in transposed contexts (Levinson, 2003; McNeill, 1992; Wilkins, 2003 inter alia). In non-transposed contexts, both egocentric and geocentric coders, when producing a direct pointing to an actual place or object, will point accurately; therefore using the 360 degree horizontal gestural space around the body. However, the main difference arises in transposed contexts.

It seems that egocentric coders mainly produce pointing in front of their body in a gestural space reduced to the visual field (see Figure 20 below). This is true also in the examination of gestures in narrations, i.e., mainly regarding metaphorical pointing (see McNeill, 1992, pp.86-92). This is unsurprising since egocentric coders constantly rely on their point of view in non-transposed condition (tending to orient themselves towards the target) but more systematically under transposition (projecting the most suitable point of view on the distant scene). Consequently, pointings are more reduced and rarely produced outside of the visual field of the speaker (Kita, 1998, 2003a; McNeill, 1992). When two speakers using an egocentric FoR produce the same gesture (say point to the right under transposition) while being face to face, their gestures will be performed as mirrored, see Figure 19a. In contrast, geocentric coders, who rely on the actual world orientation, will use the full 360 degrees of horizontal gestural space and orient their pointing in the same angular direction even when facing each other, see Figure 19b.
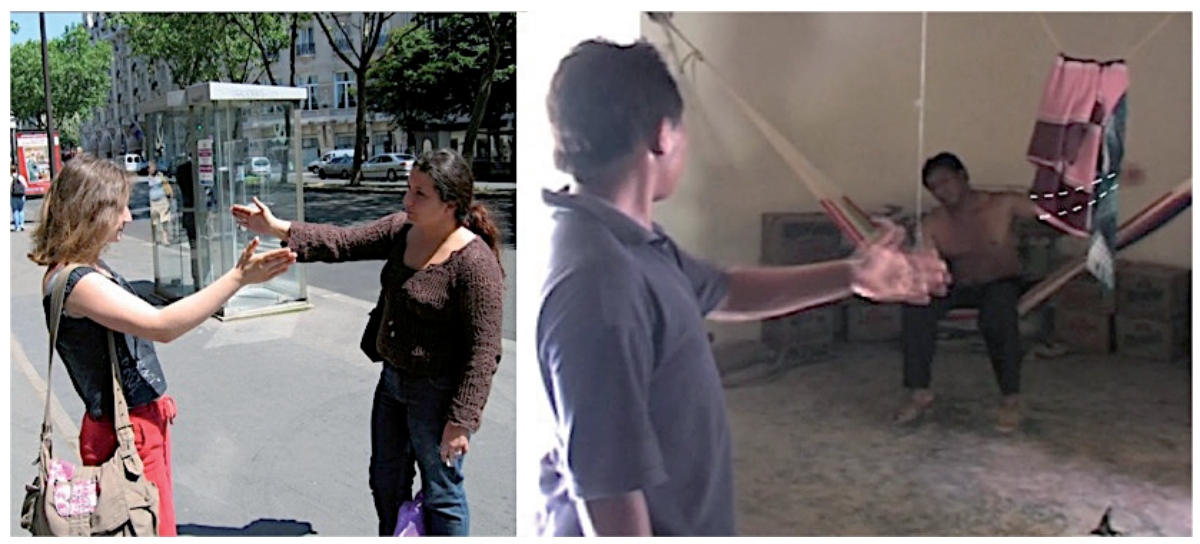

Figure 19. (a) egocentric coders pointing in the same 'right' orientation, (b) geocentric coders pointing in the same 'east' orientation (both are pointings done under transposition) 
Since geocentric coders can use the full 360 degrees of gestural space around their body, their gestures can also be larger (using the full extended arm) (Haviland 1993, 1996; Levinson, 2003; Wilkins, 2003). However, this last criterion, though it may be a tendency (Dasen \& Mishra, 2010; Levinson, 2003), is not obligatory (see example 8 and Figure 13) and should not be taken as a formal criterion to identify geocentric pointings. Enfield et al. (2007) show that the size of the gesture has often to do with the status of the information produced: when foregrounded, the gesture tends to be larger (e.g., person is indicating 'this place') while, if the information is backgrounded the gesture will be reduced (as in anaphoric pointing, see example 2).

Geocentric coders who produce direct pointing to existing places or entities rely additionally on an 'up is far' communicative rule. They make a relevant use of the vertical 180 degrees plane that starts from the feet of the speaker (the 'here') to above his head (the more 'distant, remote, unknown'). This tendency exists among Yucatec Maya speakers (see examples below and in Le Guen, 2006) and has been noted among speakers of Arrente (Wilkins, 2003), Tsotsil (Haviland, 2000), Tseltal and Guugu Yimithir (Levinson, 2003). Obviously, as pointed out by Enfield et al. (2007) for pointing size, it is the contrastive nature of the height of the gesture that makes it an analog of the actual distance indicated.

Since geocentric coders do not always mention explicitly the angular information in their speech and constantly use actual world orientation, they need, for the placement of distant entities, to specify that they are not simply indicating the (cardinal) direction in which they are pointing. From the Yucatec Maya examples, it seems that one strategy is to use an open hand shape, close to a manual classifier to represent the entity being located (consider for instance the contrast between direct pointing done with the index finger in Figure 3 with the placement of entities in virtual space done (mostly) with open hand in Figure 16 and Figure 17). Another strategy with finger pointing is to point down (i.e., towards the earth) while mentioning the figure or the ground (see Figure 17a for instance). ${ }^{9}$ Pointing towards the ground to talk about distant entities under transposition comes in opposition to the 'up is far' rule in non-transposed pointing. This contrast allows speakers to recognize that it is not the direction that is pointed at but the figure or the ground from the transposed origo, represented by the body in the speech event. ${ }^{10}$

\section{Some consequences on other related domains}

Under transposition, egocentric coders mainly use a gestural space for pointing to existing entities that is congruent with their visual field. Geocentric coders on the other hand, make a full use of the 360 degrees gestural space around their body. The difference is schematized in Figure 20. 
Egocentric coders

gesture space

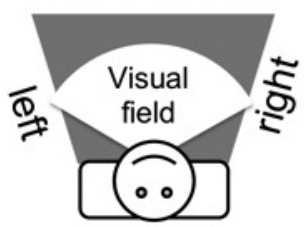

Geocentric coders

gesture space

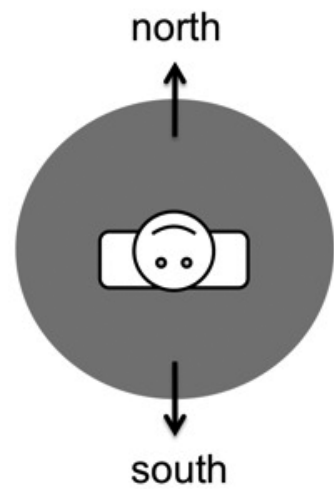

Figure 20. Zone of the gestural space actively used among egocentric and geocentric coders for pointing under transposition

Such behavior has profound consequences for the production of gesture in other domains, particularly time. Time is an abstract domain that seems to always be mapped onto space in some way. Time gestures are diverse (pointing to the sun, deictic time pointing, relational time gesture, gestures used with grammatical aspect, etc.), but their production is related to the production of spatial gestures. Egocentric speakers do not actively use the space behind their back for reference to entities in space (especially under transposition), making it available for gesturing about abstract notions. It becomes then possible to put the past to one's back in performing a point with a speech reference such as 'yesterday' or 'the other day' as in French (Calbris, 1990, p. 88), American English (Casasanto \& Jasmin, submitted), Andean Spanish (Núñez \& Sweetser, 2006) or Neapolitan Italian (de Jorio, 2000, p. 312).

For geocentric coders such metaphorical pointing is not used. Rather, the gestural mapping of time in space among geocentric coders can take various forms according to the conventions of the speech community. Among the speakers of Pormpuraawan (Australia), Boroditsky and Gaby (2010) note that the representation of time is conceived as a time flow oriented according to the cardinal directions, specifically following the course of the sun above the Earth. Pormpuraawan speakers point accordingly to the east for the past and to the west for the future. Among the Yucatec Maya, time in gestural space is organized differently. There is no mapping according to cardinal direction nor a linear conception of time flow (Bohnemeyer, 2003, 2009). An analysis of co-speech gesture in natural data and in questionnaires shows that Yucatec Maya speakers also map the gestural space for time on that for space but in a peculiar way. ${ }^{11}$ Using a geocentric FoR implies that 
any point in any direction is always by default a reference to an existing direction or an existing place identified in the speech or the context. Hence, the whole gestural space surrounding the speaker is relevant for spatial reference and only two parts of the surrounding space are co-opted for time reference. The 'now' or 'precise/specific' time is done by pointing towards the space of the feet of the speaker, i.e., mapped on the spatial 'here.' In accordance with the spatial 'up is far/remote' rule, remote time (interestingly, past or future) is gestured towards the space above the head of speaker. In this system the gesture space for past and future is collapsed in one space under the basic concept of 'remoteness.' The lack of grammatical tense in Yucatec Maya and the more general cyclic cultural conception of time facilitate this mapping (Le Guen \& Pool Balam, submitted).

The use of gestural space among geocentric coders, specifically the reluctance to use gestural space metaphorically because pointing always is taken to refer to real places could have consequences for other kinds of geocentric coders: deaf signers. In various western well-developed sign languages (American SL, British SL, NGT/SL of the Netherlands, Mexican SL), the pronominal system is based on metaphorical pointing where a region of air pointed at creates a referent (Liddell, 2003). In small-scale societies with incipient sign language (limited to an individual, a family or no more than a village), person reference is usually done by pointing to the house of the person (i.e., direct or metonymic pointing) (e.g., Sandler et al., 2011, for Al-Sayyid Bedouin Sign Language, Washabaugh et al., 1978, for Providence Island Sign Language, or de Vos, in prep., and Zeshan, 2006, p. 64, for Kata Kolok) or by using 'nicknames' or sign names (e.g., McKee \& McKee, 2000; Nyst, 2007). Meir et al. (2010) point out that a few emerging sign languages (e.g., ABSL and Kata Kolok) have been reported to use space for topographical use but not for metaphorical or grammatical use (see also Pyers \& Senghas, 2007 on Nicaraguan Sign Language). Further work is needed to describe the solutions adopted in incipient sign languages for reference in speech communities that use preferentially a geocentric frame of reference.

\section{Concluding remarks}

This article proposes a systematic framework of analysis that will permit more detailed description of frames of reference and pointing around the world. However, in contrast to studies of pointing when the egocentric frame of reference is used, when studying pointing when the geocentric frame of reference is used it is necessary for the researcher to always be able to identify the orientation of the scenes described, i.e., to have an extensive knowledge of the local geography, objects, and manmade things (roads, houses, etc.) using maps and GPS measurement. 
Gesturing that refers to space remains largely understudied. Specifically, we still have only a partial understanding of what is involved in the use of the frames of reference in spatial gesture production and the impact of this FoR preference on other related domains. The implications of choice of Frames of Reference for cognitive representation and memory has only been approached and we know virtually nothing about the impact of the preference of the FoR on gesture production among children from speech communities which use geocentric coding. Much study is needed to better understand the importance of gesture in human communication and its impact on cognition, and a closer attention to non-western data is indispensable.

\section{Acknowledgements}

I gratefully acknowledge the support of the Max Planck Society in funding this research. I would like to thank my Yucatec Maya and French participants (Dyoos bo'otik, merci!). I am sincerely indebted to the following people for helpful comments and discussions on this paper: Linda Abarbanell, Penny Brown, Kensy Cooperrider, Connie DeVos, Nick Enfield, Simeon Floyd, Susan Goldin-Meadow and her lab, Adam Kendon, Sotaro Kita, Steve Levinson, Aslı Özyürek and The Nijmegen Gesture Centre, Pamela Perniss and Lorena Pool Balam. I also like to thank the reviewers for their insightful comments.

\section{Notes}

1. The reason lies in the fact that the term 'relative' is confusing (see for instance the discussion between Levinson and Haviland in Lucy (Lucy, 1998, pp. 106-107). Any spatial localization is always done in relation to something (hence is always 'relative' to an origo). The term 'egocentric' is more explicit in referring directly to the speaker's point of view. The use of the term 'geocentric' instead of 'absolute' is motivated by the fact that etymologically, it refers to external referents and is directly contrastive with egocentric.

2. In some cases, the body of the speaker can used as the ground and therefore it would be an instance of the use of the intrinsic FoR, where the internal division of the body of the speaker would be used to locate the figure. However, these cases are not considered in this paper because in such case, the production of a pointing becomes only optional. If a speaker says "it is in front of me" it is easy to locate the target considering his or her position with or without pointing. This is not the case with the use of direct pointing where the body is not the ground but only the origo (i.e., the departure point of the vector).

3. Accuracy of direct pointings among egocentric and geocentric coders has been explored systematically (see Levinson, 2003, chap. 6)

4. In this sense, the gesture plays the role of a shifter, like 'there' (Jakobson, 1971; Silverstein, 1976). 
5. Although it is true that the gestural space is, in some cultures, already implicitly divided. In English for instance, the left-right axis allows to rank referents in a chronological order or by valence (see Casasanto \& Jasmin, submitted, 2009). I thank Kensy Cooperrider for this comment.

6. In this paper, I limit the analysis of metaphorical gesture to pointing to existing places, but see for instance Cienki \& Müller (2008) for a broader analysis of gesture and metaphors.

7. This cultural attitude could also be related to what Danziger $(2006,2010 \mathrm{~b})$ has showed among Maya Mopan, that is a reluctance to engage in fictional or abstract reasoning in general. I thank Suzanne Gaskins for this comment.

8. The author conducted an elicitation session with the speaker of the whole narration and specifically asked about this pointing.

9. Note that in Figure 16b the participant first performs a high point to indicate the north (which is actually north from his current body position), but uses an open hand form to place the second element (the GS, which is south of the shop, but not from his body's current position).

10. I am indebted to Sotaro Kita for this comment.

11. To assess gestures occurring with speech ('co-speech gestures'), four different video-recorded sessions (more than 1 hour) were examined (see Le Guen \& Pool Balam, submitted, for details). The questionnaire (non published) was run with 5 women and 2 men.

\section{References}

Bohnemeyer, Jürgen (2003). Invisible time lines in the fabric of events: Temporal coherence in Yucatec narratives. Journal of Linguistic Anthropology, 13 (2), 139-162.

Bohnemeyer, Jürgen (2009). Temporal anaphora in a tenseless language. In Wolfgang Klein \& Li Ping (Eds.), Expression of time (pp. 83-128). Berlin \& New York: Mouton de Gruyter.

Borg, Emma (2002). Pointing at Jack, talking about Jill: Understanding deferred uses of demonstratives and pronouns. Mind \& Language, 17 (5), 489-512.

Boroditsky, Lera \& Alice Gaby (2010). Remembrances of Times East. Absolute spatial representations of time in an Australian aboriginal community. Psychological Science, 21 (11), 1635-1639

Brown, Penelope \& Stephen C. Levinson (2009). Language as mind tools: Learning how to think through speaking. In J. Guo, E. V. M. Lieven, N. Budwig, S. Ervin-Tripp, K. Nakumara, \& S. Ozcaliskan (Eds.), Crosslinguistic approaches to the psychology of language: Research in the traditions of Dan Slobin (pp. 451-464). New York: Psychology Press.

Bühler, Karl (1983). The deictic field of language and deictic words. In Robert J. Jarvella \& Wolfgang Klein (Eds.), Speech, place, and action: Studies in deixis and related topic (pp.9-30). Chichester, New York, Brisbane, Toronto, \& Singapore: John Wiley \& Sons LTD.

Calbris, Geneviève (1990). The semiotics of French gestures. Bloomington, IN: Indiana University Press.

Casasanto, Daniel \& Kyle Jasmin (submitted). The hands of time: Temporal gestures in English speakers. 
Casasanto, Daniel \& Kyle Jasmin (2009). Emotional valence is body-specific: Evidence from spontaneous gestures during US presidential debates. In N. Taatgen \& H. Van Rijn (Eds.), Proceedings of the 31st Annual Meeting of the Cognitive Science Society (pp. 1965-1970). Austin: Cognitive Science Society.

Cienki, Alan J. \& Cornelia Müller (2008). Metaphor and gesture. Amsterdam \& Philadelphia: John Benjamins.

Clark, Herbert H. (1996). Using language. Cambridge: Cambridge University Press.

Danziger, Eve (2006). The thought that counts: The interactional consequences of variation in cultural theories of meaning. In N. J. Enfield \& S. C. Levinson (Eds.), Roots of human sociality. culture, cognition and interaction (pp. 259-278). Oxford \& New York: Berg Publishers.

Danziger, Eve (2010a). Deixis, gesture, and cognition in spatial Frame of Reference typology. Studies in Language, 34, 167-185.

Danziger, Eve (2010b). On trying and lying: Cultural configurations of the Gricean Maxim of Quality. Intercultural Pragmatics, 7 (2), 199-219.

Dasen, Pierre \& Ramesh C. Mishra (2010). Development of geocentric spatial language and cognition. An eco-cultural perspective (Cambridge Studies in Cognitive and Perceptual Development, No. 12). Cambridge, UK: Cambridge University Press.

Enfield, Nick J. (2009). The anatomy of meaning speech, gesture, and composite utterances. Cambridge, UK: Cambridge University Press.

Enfield, Nick J., Sotaro Kita, \& Jan Peter de Ruiter (2007). Primary and secondary pragmatic functions of pointing gestures. Journal of Pragmatics, 39 (10), 1722-1741.

Engle, Randi A. (1998). Not channels but composite signals: speech, gesture diagrams and object demonstrations are integrated in multimodal explanations. In M. A. Gernsbacher \& S. J. Derry (Eds.), Proceedings of the Twentieth Annual Conference of the Cognitive Science Society (pp. 321-327). Mahwah, N.J.: Erlbaum.

Floyd, Simeon (submitted) Grammar across modes: celestial gesture and temporality in the Nheengatú verb phrase. Max Planck Institute for Psycholinguistics.

Fricke, Ellen (2002). Origo, pointing, and speech: The impact of co-speech gestures on linguistic deixis theory. Gesture, 2 (2), 207-226.

Goodwin, Charles (2000). Action and embodiment within situated human interaction. Journal of Pragmatics, 32 (10), 1489-1522.

Le Guen, Olivier (2006). L'organisation et l'apprentissage de l'espace chez les Mayas Yucatèques du Quintana Roo, Mexique. Unpublished Ph.D. dissertation Thesis. Université Paris XNanterre, Paris.

Le Guen, Olivier (2009). Geocentric gestural deixis among Yucatecan Mayas (Quintana Roo, México). In Kostas Mylonas (Ed.), 18th IACCP book of selected congress papers (pp. 123136). Athens, Greece: Pedio Books Publishing.

Le Guen, Olivier (2011). Handling Frames of Reference: the co-dependence of speech and gesture in spatial cognition among the Yucatec Mayas. Cognitive Science, 35 (5), 905-938.

Le Guen, Olivier \& Lorena Pool Balam (submitted). The wheels of time: Time, space and cyclicity among the Yucatec Maya.

Hanks, William F. (1990). Referential practice: Language and lived space among the Maya. Chicago: University of Chicago Press.

Hanks, William F. (2005). Explorations in the deictic field. Current Anthropology, 46 (2), 191220.

Haviland, John B. (1993). Anchoring, iconicity, and orientation in Guugu Yimithirr pointing gestures. Journal of Linguistic Anthropology, 3 (1), 3-45. 
Haviland, John B. (1996). Projections, transpositions, and relativity. In John Gumperz \& Stephen C. Levinson (Eds.), Rethinking Linguistic Relativity (pp. 271-323). Cambridge: Cambridge University Press.

Haviland, John B. (2000). Pointing, gesture spaces, and mental maps. In David McNeill (Ed.), Language and Gesture (pp.13-46). Cambridge: Cambridge University Press.

Jakobson, Roman (1971). Shifters, verbal categories, and the Russian verb. In Roman Jakobson. Selected writings 2. Word and language (pp. 130-147). Den Haag: Mouton.

de Jorio, Andrea (2000) Gesture in Naples and gesture in classical antiquity: A translation of Andrea de Jorio's La mimica degli antichi investigata nel gestire napoletano by Adam Kendon, with an Introduction and Notes. Bloomington IN: Indiana University Press.

Kelly, Spencer D., Aslı Özyürek, \& Eric Maris (2009). Two sides of the same coin: Speech and gesture mutually interact to enhance comprehension. Psychological Science, 21 (2), 260-267.

Kendon, Adam (2004). Gesture: visible action as utterance. Cambridge: Cambridge University Press.

Kendon, Adam \& Laura Versante (2003). Pointing by hand in Neapolitan. In Sotaro Kita (Ed.), Pointing: where language, culture, and cognition meet (pp. 109-138). Mahwah, NJ: Lawrence Erlbaum.

Kita, Sotaro (1998). Expressing a turn at an invisible location in route direction. In E. HessLüttich, J. Müller, \& A. vanZoest (Eds.), Signs \& Space; Raum und Zeichen (pp. 160-172). Türbingen: Gunter Narr Verlag.

Kita, Sotaro (2003a). Interplay of gaze, hand, torso orientation and language in pointing. In Sotaro Kita (Ed.), Pointing: where language, culture, and cognition meet (pp. 307-328). Mahwah, NJ: Lawrence Erlbaum.

Kita, Sotaro, Eve Danziger, \& Christel Stolz (2001). Cultural specificity of spatial schemas as manifested in spontaneous gestures. In Merideth Gattis (Ed.), Spatial schemas and abstract thought (pp. 115-146). Cambridge \& London: Bradford Book, The MIT Press.

Kita, Sotaro (Ed.) (2003b). Pointing: where language, culture, and cognition meet. Mahwah, NJ: Lawrence Erlbaum.

Levinson, Stephen C. (2003). Space in language and cognition: explorations in cognitive diversity (Language, culture and cognition, 5). Cambridge: Cambridge University Press.

Liddell, Scott K. (2003). Grammar, gesture, and meaning in American Sign Language. Cambridge: Cambridge University Press.

Locker McKee, Rachel \& David McKee (2000). Name signs and identity in New Zealand Sign Language. In M. Metzger (Ed.), Bilingualism and Identity in Deaf Communities (pp.3-40) (Sociolinguistics in Deaf Communities) Washington, DC: Gallaudet University Press.

Lucy, John A. (1998). Space in language and thought: Commentary and discussion. Ethos, $26(1), 105-111$.

McNeill, David (1992). Hand and mind. Chicago: University of Chicago Press.

McNeill, David (2003). Pointing and morality in Chicago. In Sotaro Kita (Ed.), Pointing: where language, culture and cognition meet (pp. 293-306). Mahwah, NJ: Erlbaum.

McNeill, David, Justine Cassell, \& Elena T. Levy (1993). Abstract deixis. Semiotica, 95 (1/2), 5-20.

Meir, Irit, Wendy Sandler, Carol A. Padden, \& Mark Aronoff (2010). Emerging sign languages. In Marc Marschark, Patricia Elizabeth Spencer, \& Peter E. Nathan (Eds.), The Oxford Handbook of Deaf Studies, language, and education, Volume 2 (pp.267-280). Oxford: Oxford University Press. 
Núñez, Rafael E. \& Eve Sweetser (2006). With the future behind them: Convergent evidence from Aymara language and gesture in the crosslinguistic comparison of spatial construals of time. Cognitive Science, 30 (3), 401-450.

Nyst, Victoria A. S. (2007). A descriptive analysis of Adamorobe Sign Language (Ghana). Unpublished doctoral dissertation. University of Amsterdam.

Okrent, Arika (2002). A modality-free notion of gesture and how it can help us with the morpheme vs. gesture question in sign language linguistics (Or at least give us some criteria to work with). In Richard P. Meier, Kearsy Cormier, \& David Quinto-Pozos (Eds.), Modality and structure in signed and spoken languages (pp.175-198). Cambridge: Cambridge University Press.

Pyers, Jennie \& Ann Senghas (2007). Reported action in Nicaraguan and American Sign Languages: Emerging versus established systems. In Pamela M. Perniss, Roland Pfau, \& Markus Steinbach (Eds.), Visible Variation (pp. 279-302). Berlin \& New York: Mouton de Gruyter. Quine, Willard V. O. (1968). Ontological relativity. The Journal of Philosophy, 65 (7), 185-212.

Sandler, Wendy, Mark Aronoff, Irit Meir, \& Carol Padden (2011). The gradual emergence of phonological form in a new language. Natural Language \& Linguistic Theory, 29 (2), 503-543

Schegloff, Emanuel A. (1984). On some gestures' relation to speech. In J. Atkinson \& John Heritage (Eds.), Structures of social action: studies in conversation analysis (pp. 266-296). Cambridge: Cambridge University Press.

Silverstein, Michael (1976). Shifters, linguistic categories, and cultural description. In Keith H. Basso \& H. A Selby (Eds.), Meaning in anthropology (pp. 11-55) (School of American Research Advanced Seminar Series). Albuquerque: University of New Mexico Press.

Tomasello, Michael (2008). Origins of human communication. Boston: MIT Press.

Trope, Yaacov \& Nira Liberman (2010). Construal-level theory of psychological distance. Psychological Review, 117 (2), 440-463.

de Vos, Connie (in prep.). Sign-spatiality in Kata Kolok. Ph.D. dissertation, Nijmegen, The Netherlands: Max Planck Institute for Psycholinguistics.

Washabaugh, William, James C. Woodward, \& Susan DeSantis (1978). Providence Island Sign: A context-dependent language. Anthropological Linguistics, 20 (3), 95-109.

Wilkins, David (2003). Why pointing with the index finger is not a universal (in sociocultural and semiotic terms). In Sotaro Kita (Ed.), Pointing: where language, culture, and cognition meet (pp.171-215). Mahwah, N.J.: Lawrence Erlbaum Associates.

Zeshan, Ulrike (2006). The use of sign space in Kata Kolok. In Annual Report 2005, (pp. 63-64). Nijmegen: Max Planck Institute for Psycholinguistics.

\section{Author's address}

Olivier Le Guen

CIESAS, Calle Juárez 87, Col. Tlalpan, 14000 México DF

Mexico

ompleguen@gmail.com 


\section{About the author}

Olivier Le Guen is professor and researcher in linguistic anthropology at CIESAS (Mexico). From 2008 to 2010 he was a postdoc in the Language and Cognition Group of the Max Planck Institute for Psycholinguistics, Nijmegen. His work explores the ways in which culture and language can influence human cognition in the realm of social interaction. He has conducted sustained fieldwork studies in Mexico among the Yucatec Mayas and is interested in examining multimodal interactions. Recently, he has published a study on the conception of space and the production of gesture in Yucatec Maya (Le Guen, 2011). 